\section{La enseñanza del diseño para la generación de proyectos de titulación con potencial de implementación}

\author{
Design's teaching for the generation \\ of effective projects
}

\section{Resumen}

Poniendo como precedente la historia sobre la enseñanza del diseño y, según la experiencia de la autora como estudiante, profesional y docente principalmente de las materias de titulación, se coloca en mesa de análisis una serie de aspectos que han permitido que la enseñanza del diseño se transforme, convirtiéndose en una práctica más avanzada, con nuevas metodologías y conocimientos que le proporcionen un enfoque participativo e innovador, permitiéndole la ocupación de nuevos territorios y la generación de soluciones efectivas a las demandas de una sociedad y un mundo cambiante.

En este documento, se analiza esa transformación desde la aplicación de nuevas modalidades y procesos de diseño, promovidos desde las materias de Asesoría y Taller TFG de la Carrera de Diseño Gráfico de la Pontificia Universidad Católica del Ecuador y su visibilización a través de la presentación de algunos proyectos de titulación resultado de este trabajo y que al momento se encuentran en proceso de implementación, constituyéndose así en evidencia de que el diseño puede generar aportes de alto impacto para otras disciplinas y para la sociedad en general.

Palabas clave: diseño, educación, investigación, interdisciplina, innovación.

\begin{abstract}
With history as a background of design teaching. Thinking also 25 years ago and the author's experience as a student professional and teacher mainly degree matter. It gets on the analysis table a series of aspects that have allowed design education to be transformed. Becoming a more advanced practice with new methodologies and knowledge that provide a participatory and innovative approach. With a higher level of occupation of new territories and generation of effective solutions of a society and a changing world demands.
\end{abstract}

This document, analyze this transformation from the application of new design modalities and processes, promoted from the subjects of Consulting and TFG Workshop of the Graphic Design Career of the Pontificia Universidad Católica del Ecuador and its visibility through the presentation of some degree projects, the result of this work and which are currently in the process of being implemented, thus becoming evidence that design can generate high impact contributions for other disciplines as for society.

Keywords: design, education, research, interdisciplinary, innovation.

\section{Mariana Soraya \\ Lozada Mondragón}

Carrera de Diseño Gráfico de la Pontificia Universidad Católica de Ecuador (PUCE)

Artículo original Original Article

Correspondencia Correspondence mablozada@gmail.com

Financiación Fundings

Sin financiación

Recived: 2020-04-20 Accepted: 2020-10-21
Cómo citar este trabajo. How to cite this paper

Lozada Mondragón, M. S. (2020). La enseñanza del diseño para la generación de proyectos de titulación con potencial de implementación. I+Diseño. Revista Internacional de Innovación, investigación y Desarrollo en Diseño, 15, 123-138.

DOI: https://doi.org/10.24310/Idiseno.2020. v15i.8613 


\section{Desarrollo}

\section{Introducción}

Por muchos años el diseñador ha tenido que luchar con percepciones acerca de su profesión como un acto meramente estético, en virtud de ello, al momento de generar proyectos de diversa índole, ha sido convocado el momento en que el proyecto se encontraba pensado y ejecutado, únicamente para que lo convierta en algo "bonito", una percepción que data de los inicios de la profesión hace ya más de un siglo -tomando como referencia el recorrido histórico de Frascara, quien plantea el inicio de la profesión a inicios del siglo veinte-. Es así como muchos diseñadores se han enfocado en cambiar la imagen artística de la profesión y ya desde finales de los setenta, existía una leve idea de lo que el diseño podía llegar a ser, esto se evidencia en lo que decía Wucius Wong:

Ciertamente, el solo embellecimiento es una parte del diseño, pero el diseño es mucho más que eso [...] El diseño es un proceso de creación visual con un propósito [...] Una unidad de diseño gráfico debe ser colocada frente a los ojos del público y transportar un mensaje prefijado [...] En pocas palabras, un buen diseño es la mejor expresión visual de la esencia de «algo», ya sea esto un mensaje o un producto. (1979, p.9).

... el diseño ha dejado de ser una actividad meramente artesanal vinculada al buen gusto, sino que ha tomado una ruta distinta, convirtiéndose en una disciplina sostenida por bases teóricas
Así también Mazzeo presenta un breve resumen sobre los orígenes del Diseño Gráfico, donde menciona que sus primeros productos fueron generados por artistas plásticos, ahí muestra como el aspecto artístico ha estado presente de una u otra manera en muchos momentos del diseño y como este último, con el tiempo, se ha ido transformando y tornándose más funcional y adaptable a la resolución de problemas complejos de comunicación visual de diversa índole.

Lamentablemente identificar el gran potencial que tiene el diseño gráfico ha llevado bastante tiempo y a pesar de los esfuerzos, apenas en la última década ha despuntado con mayor fuerza, esto es evidente con las posturas de las nuevas generaciones de estudiosos en el diseño, como Bürdek, quien se refiere a que el diseño ha dejado de ser una actividad meramente artesanal vinculada al buen gusto, sino que ha tomado una ruta distinta, convirtiéndose en una disciplina sostenida por bases teóricas que ha logrado afectar a otros campos que en un principio se encontraban lejanos al diseño. (2003).

Así mismo, Frascara, treinta años más tarde de la afirmación de Wong, hace un recorrido histórico de la transformación del diseño e indica, que el objetivo del diseñador debe dejar de enfocarse netamente en la creación artística, sino que más bien se debe enfatizar los procesos de construcción de una comunicación eficaz, menciona también que el diseñador debe poseer criterios de selección que no se basen en preferencias personales, sino en analizar los contextos comunicacionales en los que va a operar, además se refiere al desarrollo tecnológico y a la fuerte influencia que las disciplinas de las ciencias sociales han tenido en la labor del diseño. (2012).

\section{Una nueva forma de ver el Diseño}

Con este precedente es importante analizar algunos aspectos que hasta la fecha siguen aquejando a las aulas de diseño, uno de ellos, que si bien es 
cierto por un lado ha determinado el avance del diseño, por otro, de forma contradictoria en muchas ocasiones lo ha estancado, me refiero a la tecnología, una herramienta que, si se lo utiliza de forma efectiva, tiene la capacidad de reducir potencialmente el tiempo de trabajo y facilita la generación de propuestas con la más alta calidad, el problema radica cuando a esta herramienta se la considera el pilar del diseño, cuando el ejercicio de la disciplina se enfoca en que tan bien se maneja un software y no en que tan efectivo es el mensaje que se construye a través de la herramienta gráfica.

Por otra parte, aunque se puede decir que la forma de hacer diseño ha cambiado sustancialmente, aún hay varios aspectos en los que se debe trabajar, y como lo menciona Yedaide en su presentación de las III Jornadas de Investigadores en Educación de la UNMDP, son situaciones que afectan a cualquier disciplina que genere una práctica de procesos proyectuales. Ella habla sobre la contraposición entre la rigidez de las prácticas actuales y el carácter dialógico que favorece los procesos de cocreación, así como el condicionamiento de las configuraciones didácticas de los proyectos de diseño, e indica que este tipo de problemáticas no se desvincula del escenario donde -cita a Mazzeose promueve la enseñanza más informativa que formativa y que «aleja a los eventuales graduados de las posibilidades concretas de asumir responsabilidad social en actos de transformación social» (2016, p. 2).

En su presentación Yedaide se refiere también a la selección de proyectos de carácter meritocrático que terminan favoreciendo a los ya socialmente favorecidos, comenta también sobre la importancia de considerar lo que llama «la conciencia de los retos de la creación» o en otras palabras la incertidumbre que implica para el estudiante la generación de nuevos productos y los riesgos a los que se enfrenta cuando se dispone a «aprender lo que no puede ser siquiera imaginado o previsto de antemano». (2016). Afirma además que «si la enseñanza no es capaz de despertar hacia lo inédito, de servir de trampolín desde lo heredado a lo aún no concebido... es difícil sostener algún valor real para la educación superior» (2016, p. 2).

Además, otro problema al que se enfrenta un proceso proyectual es el dilema de hasta que punto es posible adquirir y otorgar libertad para propiciar la inquietud por aprender y la generación de espacios para el misterio y el impulso voluntario hacia el aprendizaje, y en que instante es necesario acogerse a un proceso educativo predefinido, pero como lo indica la misma Yedaide "No es posible ni deseable, así, reducir el aprendizaje a un número de contenidos sin crear las situaciones en que estos estallan en problemas, preguntas y misterio. De otra forma, el conocimiento deviene mercancía inerte, se repliega en su nueva irrelevancia y se circunscribe en un acto de cumplimiento y adhesión a las convenciones» (2016, p. 7), además que es innegable que cada proceso posee su propia dinámica y formas de actuar.

Esto se puede complementar también con lo que comenta Forner sobre el racionalismo innato de los métodos de diseño y las aportaciones del diseñador. Ella asegura que se ha llegado a un equilibrio «los teóricos actuales defienden un espacio común a ambos conceptos, una zona híbrida entre razón e imaginación, entre metodología estricta y libertad creativa, entre ideas objetivas y 
universales y el enfoque individual de cada diseñador y cada proyecto concreto» (p. 155). Además, menciona que el diseño actual debe considerar también nuevas dinámicas que contemplan la formación de equipos de trabajo, la colaboración en redes y las estrategias transversales.

Bajo este esquema, hay otro problema inserto, pues muchos de los estudiantes enfrentan el miedo a la ignorancia, un comportamiento propio de la educación reglamentaria y tradicional, concibiéndola como una característica negativa que se debe ocultar para no quedar mal, cuando en realidad sería más fructífero que tanto docentes como estudiantes lo asuman como una oportunidad de aprendizaje, considerando que ese puede ser el punto de partida de la exploración, planteándose retos y generando interrogantes que después de la investigación y reflexión de otros, darán lugar a la generación de criterios propios y en muchas ocasiones al desarrollo de soluciones desde nuevas visiones. Del punto de vista del desarrollo de competencias que les permitan a futuro desarrollar nuevas estrategias y mejores metodologías, dominando no solo la parte conceptual, sino también sabiendo como aplicarlo a situaciones reales y concretas. Como lo expresa Forner, el docente «debe animar al alumno a perder el miedo al error. El trabajo del creador de mensajes visuales es arduo y lleno de trampas, y el peor de los enemigos al que puede enfrentarse es el temor a la equivocación, sensación que puede llegar a ser paralizante y tremendamente negativa para el estudiante» (p. 178).

Este escenario es fundamental considerarlo, más aún sabiendo que cualquier decisión de diseño se sustente en evidencias que se obtienen de la investigación, las que se encuentran tanto en los estudios teóricos de la disciplina que se han fortalecido por varios años, como desde un estudio de campo profundo y bien estructurado del entorno y sus actores, además que resulta esencial considerar lo que puntualiza Forner en cuanto a que «los conocimientos teóricos y técnicos concretos que se deben impartir, los diseñadores consideran que deben estar basados en la realidad profesional y no desde una visión subjetiva del profesor" (p. 182) o incluso bajo modas o tendencias, pues como indica Bellucia, el trabajo del diseñador se debe condicionar por el contexto social, por las realidades de sus públicos y emisores - pues él es solo un intermediario-, por códigos de comunicación bien establecidos y no por su gusto, estilo personal o por modas (2007). Es así como al considerar todo esto, se abre un abanico de posibilidades al diseñador, puesto que le permite cotejar información mucho más compleja y determinar con criterio el camino más efectivo hacia la generación de soluciones para necesidades específicas, sin tener limitantes propias o externas que les resten potencia a sus planteamientos gráficos.

Asimismo, otro aspecto que también se analiza ya desde los años 90, es lo que comenta Forner, cuando se refiere a que «El diseñador, al afrontar, plantear y actuar frente a un problema concreto, no sigue ninguna metodología lineal que pueda ser circunscrita a un ámbito científico concreto, sino que trabaja mediante estructuras mentales y estrategias creativas mucho más complejas de lo que hasta esos momentos se pensaba en el mundo académico» (p. 154), esto le permitió al diseñador desligarse de estructuras predeterminadas de investigación y empezar a explorar nuevas formas que se ajusten con mayor efectividad a sus necesidades de búsqueda de información. 
Esto ha dado lugar a que la academia del diseño ya no se conforme con tomar los procesos investigativos generales o los construidos desde otras áreas del conocimiento, sino que se enfoque en generar sus propias metodologías; $y$, en los últimos años, combine metodologías propias con insumos metodológicos e investigativos de apoyo tomados de las áreas del conocimiento específicas vinculadas a cada proyecto.

Para ello es fundamental trabajar de una manera planificada y sistemática, puesto que cada paso es un determinante para el resultado final y la sistematización facilita la identificación de conexiones no solo entre problemas existentes sino también entre actores; optimiza recursos, pues permite identificar con facilidad aquellas herramientas que ya se han utilizado y pueden ser adaptados en situaciones actuales; además que le permite ver con mayor claridad y orden el panorama del problema al que se enfrenta, presentándole al mismo tiempo las posibles oportunidades de intervención del diseño.

Es así como ya desde los 90 se pretende establecer lazos más estrechos entre la investigación y la interdisciplina, así lo indica Ander-Egg en su libro El taller una alternativa de renovación pedagógica, donde explica sobre la importancia de profundizar en lo interdisciplinar, indicando que es fundamental la generación de un pensamiento y abordaje sistémico, donde se consideren cuatro aspectos importantes:

1. Manejar métodos de investigación que aborden la realidad con un enfoque holístico.

2. Que las formas de pensar se vinculen a entender las interacciones los problemas y la resolución de estos considerando los diversos sectores de intervención.

3. Utilizando metodologías de diseño que posean un carácter globalizador e incluyan planes y estrategias.

4. Que posean un marco de referencia común que identifiquen similitudes desde las diversas disciplinas.

También sugiere que uno de los principios pedagógicos del taller es integrar a la docencia, investigación y práctica en un solo proceso o proyecto de trabajo donde docentes y alumnos participen activa y responsablemente y donde consideren además la exigencia del conocimiento de la realidad sobre la que se va a actuar. (1991).

Esto evidentemente se vincula con el contexto social y quienes lo conforman, por lo tanto, es trascendental que el diseñador se inserte en el entorno de trabajo y lo comprenda, de tal manera que pueda brindar soluciones más adaptables a esa realidad, un mensaje que se aborda ya desde los 80 con autores como Ricard o Jones, quienes afirman que no se puede realizar diseño si no se vincula directamente con la persona a quien se pretende satisfacer y que se conciben respuestas proyectuales más pertinentes cuando estas se generan a partir de la realidad y las necesidades de la gente. Claro está, eran afirmaciones que en muchos casos se enfocaban en la persona que solicitaba el diseño, más no siempre en el usuario final (1986), y como lo confirma Frascara en la actualidad "Los diseñadores debemos entender las necesidades de la gente,
... es fundamental la generación de un pensamiento y abordaje sistémico, donde se consideren cuatro aspectos importantes 
... en las dos últimas décadas

la profesión se ha consolidado de forma impactante, fundamentándose en una fuerte reflexión teórica, un trabajo profundo de investigación y una práctica proyectual sus deseos, sentimientos, expectativas, posibilidades y limitaciones, preferencias y conductas, y, además, sus contextos. Esto debe incluir las cosas que ven, la estética que prefieren, las cosas que leen, y los medios de información que usan» (Frascara, 2017, p. 10).

Frascara enseña que el diseño debe centrarse en el usuario, considerándolo como el centro de su labor y la persona que interactúa con las producciones de diseño; por lo tanto, este diseño debe enfocarse en sus necesidades, dejando de lado la visión que se tenía hace algunos años, donde el diseño se centraba en satisfacer las necesidades comerciales de las empresas u organismos contratantes o emisores de la comunicación, logrando con ello que el diseño gane mayor fuerza no solo en el ámbito comercial y económico sino también en el social, cultural, como lo indica Mazeeo:

"La complejización de los espacios urbanos y la creciente necesidad de comunicar a mayores e indiscriminados grupos sociales con la consecuente dificultad para hacerlo con los métodos tradicionales del pregón y la comunicación interpersonal, impulsó el desarrollo de una práctica que ya venía realizándose desde tiempo atrás, aunque sin formalizarse en un campo específico. Cualquiera que fuese el origen de las prácticas que hoy conocemos como Diseño Gráfico, no hay duda de su fuerte y particular relación con los cambios sociales, culturales y económicos» (2017, p. 37) y en el entorno actual aún en áreas que hace algunos años hubiesen resultado inimaginables.

A su vez y respecto a la enseñanza del diseño y su proyección hacia el futuro, Cecilia Mazzeo confirma que en las dos últimas décadas la profesión se ha consolidado de forma impactante, fundamentándose en una fuerte reflexión teórica, un trabajo profundo de investigación y una práctica proyectual que junto a otros profesionales genera soluciones efectivas para las necesidades de las personas para quienes está destinado el producto, pero que a pesar de ello sigue luchando para terminar de una vez con la visión apenas decorativa que por muchos años la sociedad ha tenido de ella reflexionando sobre una nueva forma de saber hacer diseño (2015).

En este punto, quizá lo que necesitamos es estrechar aún más esos lazos con otras disciplinas mediante la investigación profunda y la generación de soluciones gráficas sustentadas no solo en teorías del Diseño, sino también en estudios de otras áreas del conocimiento, y ante ello el proceso de enseñanza-aprendizaje se enfrenta a otro reto, la percepción de varios estudiantes quienes mencionan que la investigación y consulta de libros es infructuosa, pero quizá eso se debe a la forma en que se generan estos procesos, pues como señala Finkel, una asignatura que se centra en la indagación no puede enfocarse en el contenido tradicional, sino en dar respuesta a un problema o pregunta, sabiendo que si los estudiantes están interesados en la indagación, tendrán mayor impulso para continuar en la búsqueda. (2008), este mismo autor comenta: "Si toda la asignatura es concebida como una indagación -la investigación de un asunto o problema-, entonces leer y discutir libros con un espíritu indagador no destacarán como actividades inusuales» (2008, p. 103). 
Es evidente la importancia de la riqueza que implica la relación con otras disciplinas, y que mejor explicado que la cita que Yedaide hace de Szturwark "Construir conocimiento implica construir relaciones» (2015, pp. 57-58), por ello es fundamental que desde la academia se siga buscando la participación del diseño en proyectos interdisciplinares de mayor impacto donde se reposicione al diseñador y se logre transformar la percepción del mismo, de tal manera que en algún momento el diseñador llegue a tener el mismo nivel de importancia que el resto de profesionales que participen en el proyecto, como cita Tresserras a Martín en su artículo Diseño e interdisciplinariedad. Una visión «El pensamiento simple no basta para resolver cabalmente un problema; es necesario el ejercicio del pensamiento complejo que incluye la estrategia y el trabajo transdisciplinario" (2015), es así como, en la academia no es posible desligar la resolución de problemas de diseño de la participación interdisciplinar, como lo expresa Finkel «La enseñanza centrada en problemas es interdisciplinar por naturaleza debido a que se centra en una pregunta, y las preguntas más interesantes pueden ser examinadas con utilidad desde la perspectiva de múltiples disciplinas» (2008, p. 124).

Finalmente, uno de los grandes logros de los últimos años, es que el diseñador comprenda que su trabajo no termina el momento en que se entrega el producto, sino que el diseño es cíclico y requiere de una validación, seguimiento y feedback continuo, puesto que esto permite que cada diseñador repiense su propuesta, reflexione e investigue sobre las posibles mejoras.

A partir de lo mencionado, es trascendental que desde los primeros años de estudio el estudiante se vea enfrentado a problemáticas reales y que acorde a su nivel de conocimiento de la disciplina vaya proponiendo soluciones de diseño donde el usuario sea siempre el centro de su labor, buscando que el estudiante desarrolle criterio propio sobre sus decisiones, lo que a futuro le servirá para desenvolverse con solvencia en la vida profesional.

Todo esto requiere también que la figura del docente cambie, puesto que ya no caben los discursos complejos e interminables, la posesión de la verdad absoluta y un diseño basado en supuestos y en ejemplos dados a través de los textos nada más; ahora, considerando que el diseño es una disciplina proyectual es fundamental que el docente genere un discurso más fluido y comprensible, que enfoque sus ejemplos en propuestas reales y actuales, que genere trabajos que los pueda realizar junto a sus estudiantes, planteando siempre un objetivo y proponiendo una metodología clara y una investigación interdisciplinar y como dice Litwin «Quizás, el mayor desafío para los docentes es encontrar la adecuación del problema a las posibilidades cognitivas de sus estudiantes: ni tan simple como para que lo desechen ni tan complejo como para desanimarlos. En relación con ellos, esta estrategia permite que encuentren con facilidad la relación de los conocimientos científicos con la vida real» $(2012$, p. 99).

Es fundamental además que ahora el docente se convierte en un asesor, canalizador, un guía que al mismo tiempo deba preocuparse por mantenerse actualizado la práctica profesional y en las actividades investigativas, más aún si es capaz de insertar estas prácticas en los procesos de trabajo proyectuales 
... es importante analizar también la forma en que todo lo mencionado desde la teoría se ve reflejado en la práctica académica del día a día dentro del aula, llegando con ello en el mejor de los casos a poner en práctica los diversos modos de investigación que cita Rodríguez según lo que describen Poggenpohl y Sato (2003): investigación empírica, investigación teórica e investigación metodológica, así como la aproximación humanística, que Margolin lo presenta como «una práctica social donde es fundamental considerar el contexto en el que ocurre el diseño, la situación social de la que surge la acción humana» (2016).

Lo mencionado se puede cotejar con lo que expresa Mazzeo al hablar sobre Diseño y sistema:

La inscripción en el campo de las disciplinas proyectuales supone pensar al Diseño Gráfico como una práctica reflexiva que involucra procesos metodológicos, analíticos, decisionales y propositivos; instancias que tienen características particulares siendo su mayor diferencia la naturaleza en la cual se despliegan.

En todas las disciplinas proyectuales, las instancias de investigación, análisis y fundamentación conceptual se expresan en términos textuales mientras que las propuestas de resolución lo hacen en términos formales (2017, p. 23).

Como se puede ver, existe una serie de aspectos que se han ido trabajando durante años en el ámbito académico, procurando que esa práctica reflexiva de la disciplina se inserte cada vez con mas fuerza en cada una de las actividades que se generan en las aulas y se proyectan hacia la actividad profesional; en este punto, es importante analizar también la forma en que todo lo mencionado desde la teoría se ve reflejado en la práctica académica del día a día.

\section{Aplicación de las nuevas modalidades de aprendizaje del Diseño en los pro- yectos académicos de titulación}

Ahora, desde la experiencia en otras universidades y en la Pontificia Universidad Católica del Ecuador (PUCE), específicamente en el acompañamiento para el desarrollo de los proyectos de titulación, se ha podido ver una profunda transformación en las propuestas estudiantiles, puesto que poco a poco se han ido insertando los aspectos antes mencionados.

Es claro que hace aproximadamente diez años, el desarrollo de un proyecto de titulación llevaba casi tres años desarrollarlo, a veces más y en ocasiones se ha visto estudiantes quedándose en el final del camino hacia el ámbito profesional, esto debido a que el alumno llegaba a los últimos niveles con una formación basada en los orígenes del diseño, llenos de teoría y escasa aplicación de sus saberes en propuestas reales, lo que ocasionaba que en la generación del proyecto de titulación se luchaba porque el estudiante entienda que ningún diseño sale de lo que uno desea hacer, sino que se origina en la identificación de una necesidad o un problema, los que luego eran identificados de forma general y sin criterio sobre cuáles de ellos podían ser solucionados por el diseño y cuáles no. Además de que no existía una formación investigativa y grandes falencias en la ejecución tecnológica de la profesión.

Tiempo más tarde, las falencias tecnológicas se redujeron exponencialmente, puesto que se evidenció los grandes problemas suscitados debido a la brecha generacional entre docentes y estudiantes y los grandes avances tecnológicos 
generados en los últimos años, lo que ha demandado una actualización continua de los profesores, así como de los mismos estudiantes.

Respecto a la fase investigativa, ha llevado un poco más de tiempo y es un tema que se sigue transformando, ya que, si bien es cierto, desde siempre se ha investigado, el enfoque y profundidad ha ido mejorando con el pasar del tiempo.

En cuanto al trabajo interdisciplinar, es una puerta que se la ha ido abriendo en los últimos años, pero que falta aún mucho espacio por explorar y ganar.

A la fecha, es posible afirmar con mucho agrado que los proyectos de titulación de Diseño Gráfico de la PUCE han mejorado exponencialmente, pues si bien es cierto, por ya varios años la Carrera estableció que los proyectos de titulación se realicen durante el último año de estudio, es decir séptimo y octavo nivel, los estudiantes no lo lograban, teniendo que extenderse casi un año más e incluso algunos se quedaban en el camino. Ahora ya se tienen estudiantes que casi al mismo tiempo que terminan sus niveles de estudios, se encuentran a un promedio de cuatro a nueve semanas de su graduación, y esto incluso debido a temas de revisión, evaluación y administrativos, siendo el propósito final que los próximos semestres el estudiante culmine su última semana de clases con la defensa de su trabajo de titulación y egrese con su título en mano.

Por otra parte, en estos nuevos proyectos, ya se pueden encontrar textos que evidencian un trabajo de investigación más amplio y que se apoya en el conocimiento de otras disciplinas, soluciones mejor fundamentadas y ajustadas a la realidad actual, así como productos de alta calidad que responden a una problemática específica, que han sido comprobados y que en ciertos casos son parte de un trabajo en equipo con otras carreras como aportes a procesos de investigación.

En cada uno de estos procesos se ha dejado de lado la aplicación de una metodología específica y las directrices del docente en la toma de cada una de las decisiones de diseño, sino que en estas materias es totalmente evidente la función del docente como un tutor, pues como mencionan Branda \& Porta (2012), las figuras cambian de verdugos a entusiatas, de aprender lecciones a la generación de ambientes de aprendizaje crítico y natural, de una linealidad disciplinar al descubrimiento de conexiones multidisciplinares, del trabajo con lo cercano conocido a la construcción de nuevos conocimientos ligados al misterio y lo ajeno, de la insensibilidad técnico-racional a una mentalidad abierta a través de los sentidos, de dejar de lado la enseñanza tradicional para estimular a los estudiantes para que reconstruyan las realidades.

Es así que todo esto se trata de aplicar en los procesos de titulación, pues cada estudiante realiza su propio proceso de investigación, definiendo a través de autores la metodología de diseño que más se ajuste a su problema y adaptándola en caso de considerarlo necesario, él mismo define las herramientas que le servirán para el análisis teórico y de campo, adquiere la capacidad de vincularse con otras disciplinas, comprender sus diversas posturas y es capaz de reflexionar y definir los aspectos más relevantes para la toma de decisiones, así como identificar con claridad las necesidades de su público objetivo, luego 
genera el concepto de diseño, ejecuta la propuesta y comprueba a través de especialistas del diseño, de las otras disciplinas participantes, de las entidades o instancias requirentes y del mismo público objetivo que el objetivo de diseño se haya cumplido; todo esto acompañado del docente cuya función principal es darle pequeñas pautas sobre los diversos caminos que pudiese tomar y enfrentarlo al panorama que tendría al tomar cada uno de ellos, fomentando con esto que los estudiantes generen mayor criterio y puedan asumir la responsabilidad de las decisiones tomadas.

Por otra parte, como mencionan De Santis \& Yedaide en su artículo, «el trabajo cooperativo es valorado también desde las perspectivas de lo que suele conocerse como didáctica general. En esta línea de trabajo, Camilloni (2010) plantea que, a través de la discusión con sus pares, los alumnos logran hilar diferentes razonamientos y adoptar puntos de vista distintos. A partir de estas perspectivas diferenciales, les es posible juzgar e intercambiar argumentos, así como justificar sus propias posiciones y decisiones», lo que claramente enriquece el conocimiento no solo propio sino también el que se genera a partir de las experiencias del otro.

Además, es bueno ver que la apuesta por una nueva forma de hacer diseño ha generado resultados de alto impacto tanto dentro como fuera de la institución, puesto que varios han ganado concursos, se están implementando o se encuentran en proceso de implementación. A continuación, se presentan algunos ejemplos que reflejan el trabajo desarrollado en los últimos semestres en los que se han aplicado estos ajustes a los procesos de titulación:

1. Viamiguitos fue el ganador del Reto Construye Ciudad 2019. Es un proyecto que busca generar un cambio positivo en la cultura vial ciudadana desde la etapa inicial con normas básicas de seguridad y valores ciudadanos. Al momento se encuentra en negociaciones para ser implementado en las escuelas municipales del Distrito Metropolitano de Quito (fig. 1).

2. Memo fue el proyecto ganador del concurso CITI microemprendedor del año. 2019. Es un material lúdico para aplicación profesional en las terapias de memoria de trabajo para niños de 6 a 8 años que padecen trastorno por déficit de atención e hiperactividad (TDAH), listo para producción y venta a centros terapéuticos del país (fig. 2)

3. Re-conectar fue uno del los proyectos que obtuvo mención en la Bienal Iberoamaricana de Diseño 2019. Es un material lúdico que apoya en el tratamiento de personas con Alzheimer en etapa leve y moderada, el que en 2019 ya tuvo su primer tiraje de 100 unidades, el que fue vendido en el $1^{\circ}$ congreso de Alzheimer en Ecuador (fig. 3).

4. Savia es un material gráfico informativo que busca incentivar la práctica de lactancia materna exclusiva tomando como caso de estudio la sala de lactancia de la Pontificia Universidad Católica del Ecuador, lugar en el que el proyecto ya se encuentra implementado y que se encuentra en negociaciones en otras instancias públicas y privadas vinculadas a la Red de Investigación de Primera Infancia PUCE (Red PI PUCE) (fig. 4). 


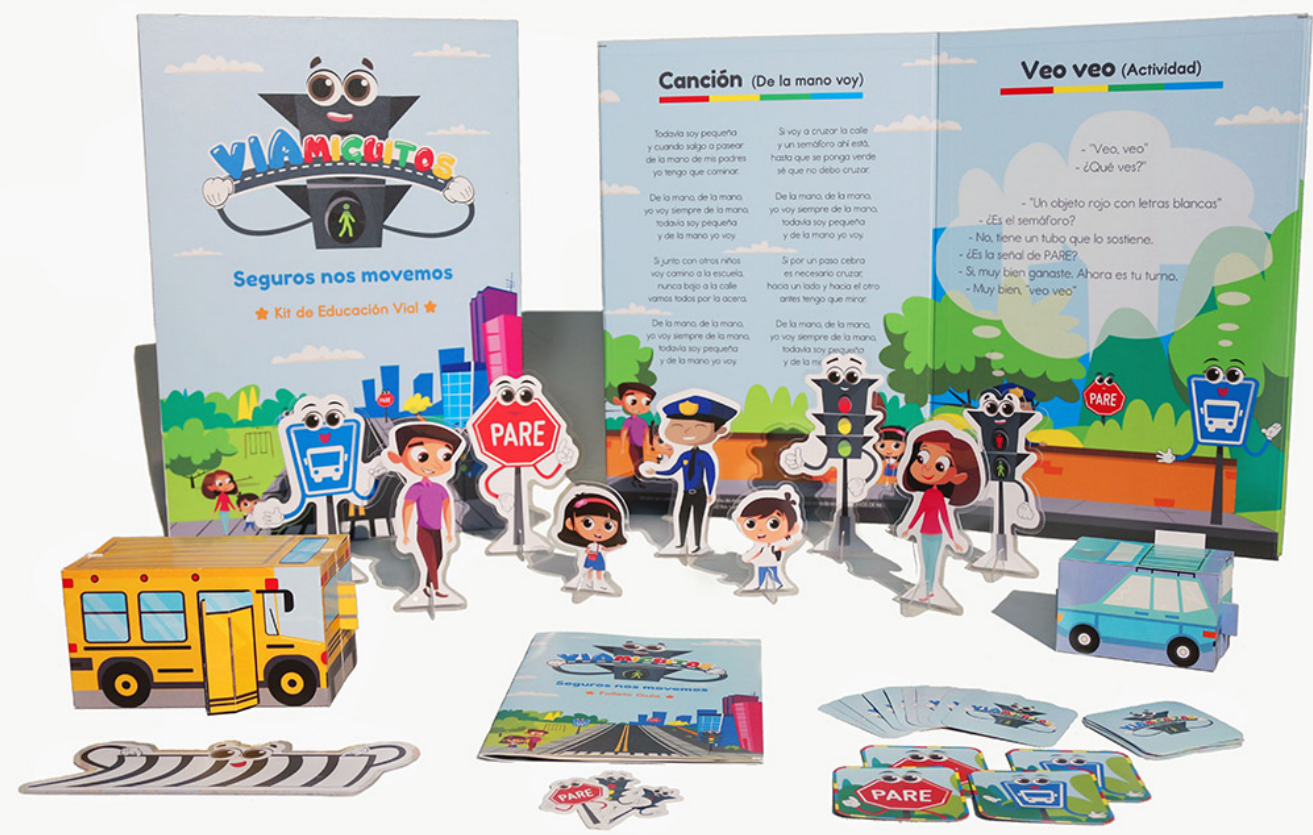

Fig. 1. TFG Jessica Arellano. Producto: Viamiguitos.

Fig. 2. TFG Scarlett Vera. Producto: Memo.
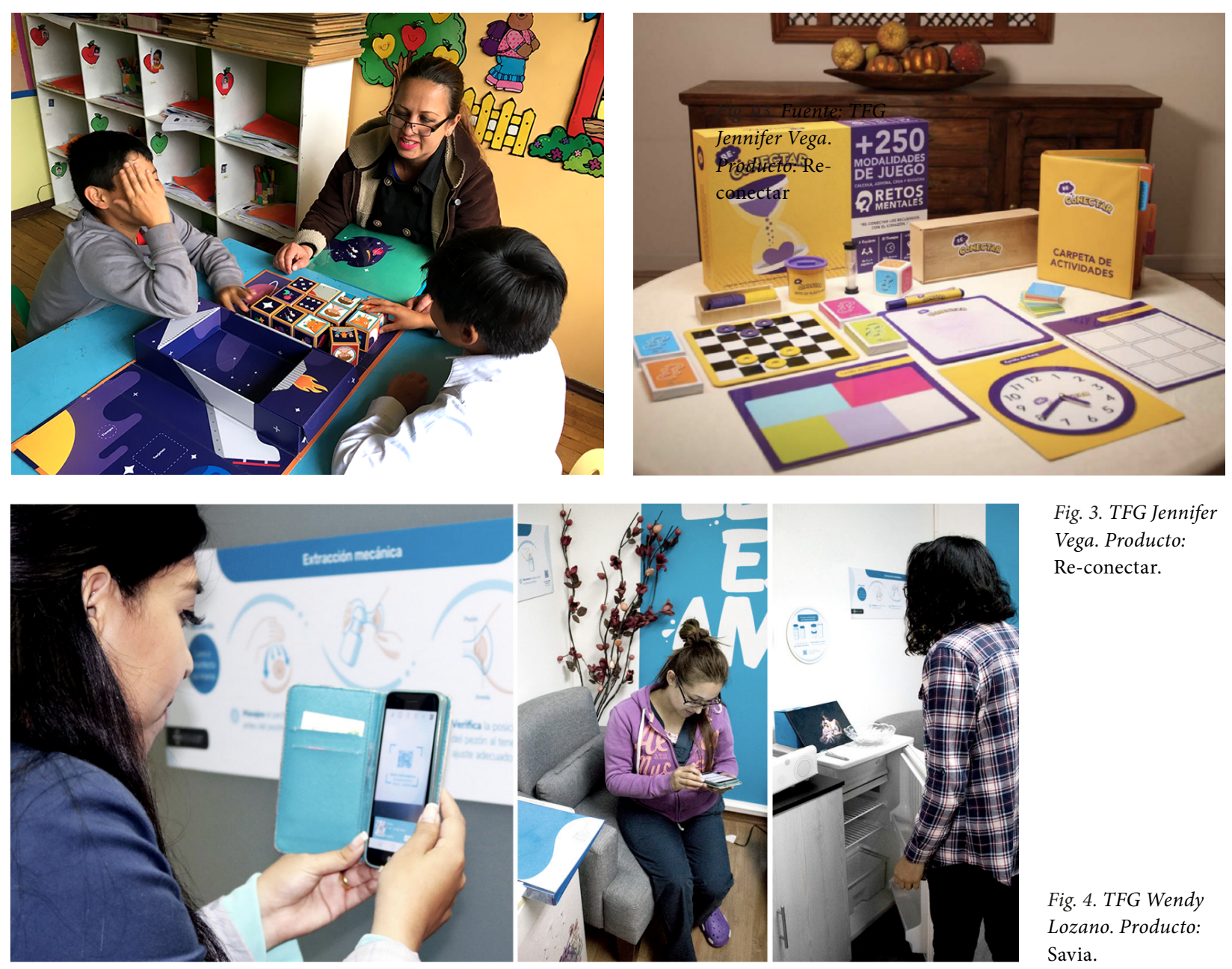

Fig. 3. TFG Jennifer Vega. Producto: Re-conectar.

Fig. 4. TFG Wendy Lozano. Producto: Savia. 


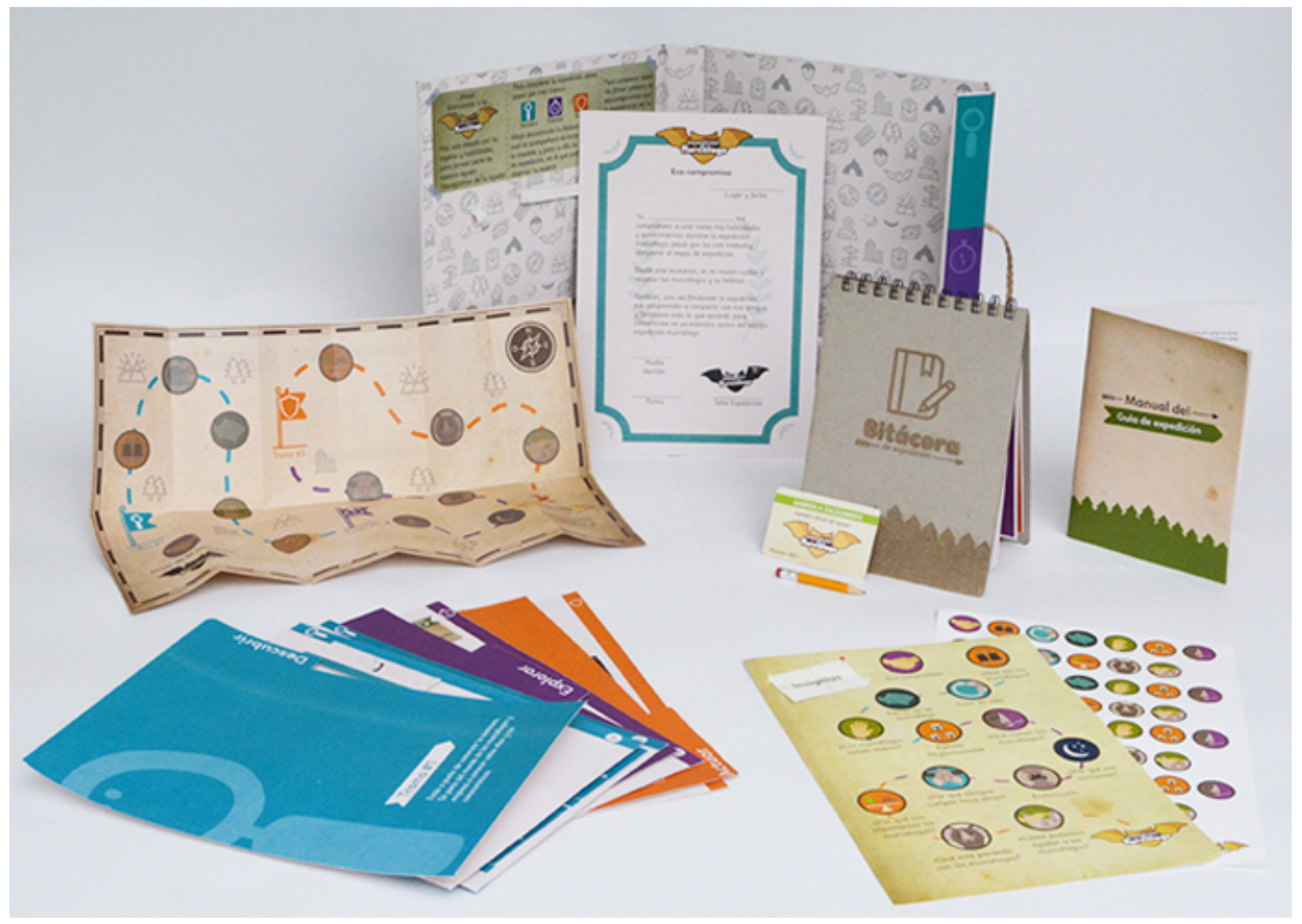

Fig. 5. TFG Gabriela Zaldumbide. Kit Expedición murciélago.
Otro de los ejemplos exitosos que posee la Carrera de Diseño de la FADAPUCE, es Expedición murciélago, un proyecto de titulación cuyo objetivo se enfoca en resolver una problemática de educación medioambiental no formal que se identifica a partir de una investigación conjunta entre las Carreras de Biología y Diseño Gráfico denominada Diversidad, distribución, filogenia, conservación y diseño de recursos educativos sobre los murciélagos de hoja nasal (Phyllostominae: Phyllostomidae) ${ }^{1}$ en Ecuador.

Este proyecto fue generado como parte de los procesos de investigación del equipo del Museo de Zoología de la Pontificia Universidad Católica del Ecuador (PUCE), en este caso a cargo de María Alejandra Camacho, y de la participación de la Carrera de Diseño gráfico a través de la docente Mariana Lozada y la estudiante Gabriela Zaldumbide, quien además de aportar en el proceso investigativo, abordaría una de las necesidades gráficas identificadas para el desarrollo de su proyecto de titulación.

1 Es una especie rara por naturaleza y muy poco representada en las colecciones científicas del país. En el Ecuador es considerada como Vulnerable debido a los pocos registros que se tienen de su presencia. Además se estima que sus poblaciones presentan una declinación continua a causa de la deforestación que sufren los bosques húmedos tropicales del noroccidente del país. 

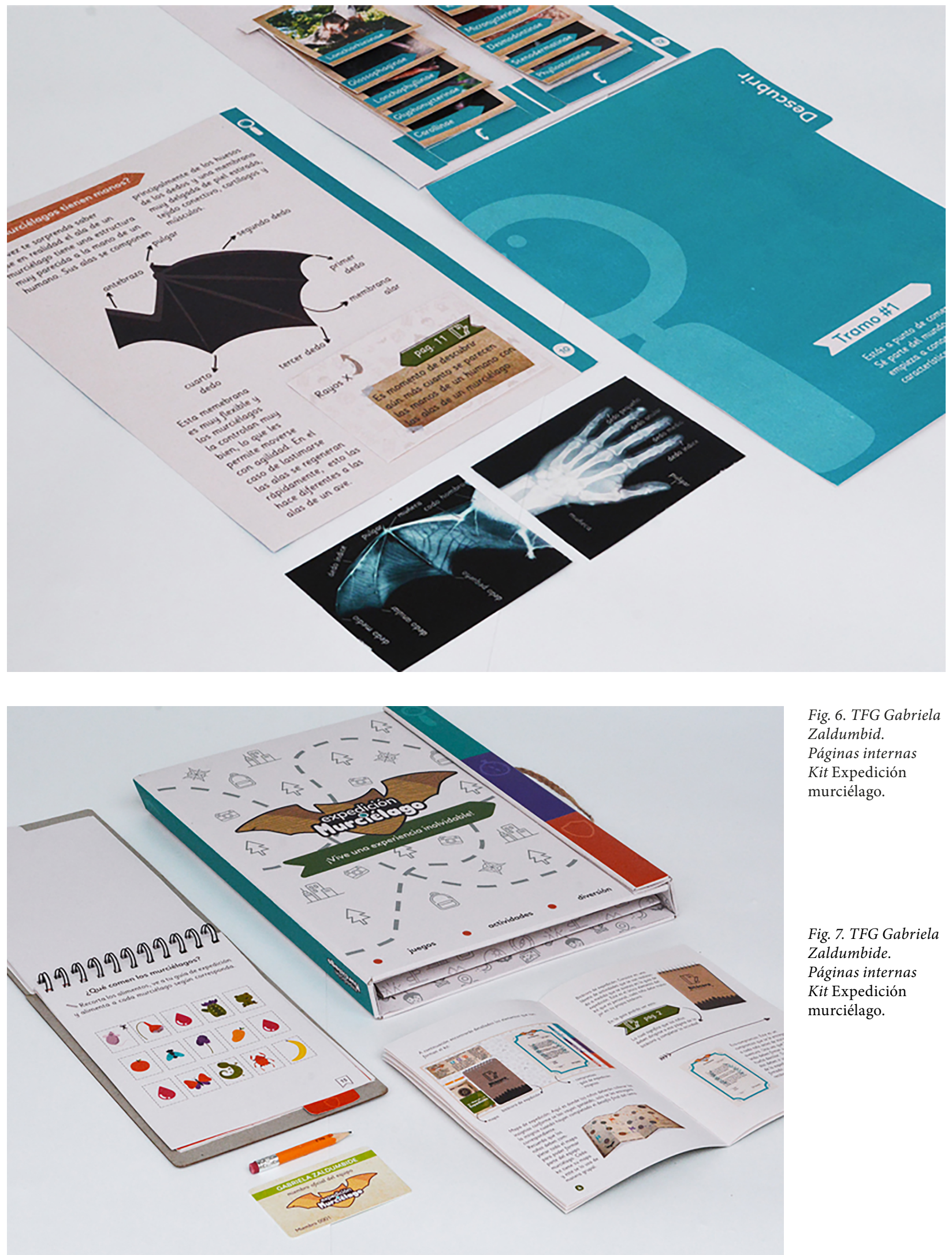

Fig. 6. TFG Gabriela

Zaldumbid.

Páginas internas

Kit Expedición

murciélago.

Fig. 7. TFG Gabriela

Zaldumbide.

Páginas internas

Kit Expedición

murciélago. 
En este proceso se considera además el cumplimiento de los principios dados por el Designing for childrens
Durante la ejecución de esta investigación se puso en clara evidencia la realidad del diseño en el campo académico y profesional, puesto que la iniciativa parte de un primer contacto en la ejecución de proyectos de servicio comunitario entre ambas carreras, luego se consolida a partir de que la investigación que nace como una propuesta de biología, requiere contar con la participación de otra disciplina para ser aprobada, es ahí donde se inserta a la carrera de Diseño, mirándola como una alternativa de aprobación del proyecto y una opción para generar material educativo de apoyo con mayor efectividad.

En este punto, es fundamental recalcar el aporte de los documentos generados por la North American Association for Environmental Education (NAAEE), Universidad de Florida y Cornell University, a través de los cuales se encontraron las pautas interdisciplinares para abordar el problema, así como las consideraciones pedagógicas y los procesos que contribuyeron para ajustar la metodología de diseño aplicada y avisorar una posibilidad de inserciones más profundas del diseño en los procesos de educación medioambiental no formal.

Respecto al proceso de titulación en sí, la estudiante tomó como eje lo que menciona Novo (2005), «actualmente este tipo de educación representa uno de los ejes de cambio y transformación social más importantes. Y entre sus características están que: es una educación contextualizada, favorece los procesos interdisciplinares, permite que surja la conciencia participativa, flexibiliza el papel del profesor y alumno, estimula las relaciones entre educación y trabajo, usa múltiples recursos y vías para el aprendizaje, y estimula la creación de redes». Ante ello estructura su investigación con bases teóricas de Biología, Educación, Psicología y claro está Diseño, y aplica el Design Thinking con ciertas inserciones ligadas a los procesos de educación como la Guía del flujo de desarrollo de programas desarrollada por la Asociación Norteamericana de Educación Ambiental (NAAEE), la que propone los pasos a seguir para la buena y eficaz construcción de programas, y plantea las pautas para la excelencia en materiales de Educación Ambiental. En este proceso se considera además el cumplimiento de los principios dados por el Designing for childrens.

El producto de titulación resultante de este primer acercamiento del Diseño a la educación medioambiental no formal, específicamente a la preservación de los muerciélagos de hoja nasal, comprende el diseño de material de educación medioambiental no formal dirigido a niños de 7 a 9 años que informe y fomente la conservación de los murciélagos de hoja nasal, mediante un producto lúdico didáctico que, a través de la aventura, acerque al niño a los entornos naturales en los que vive el animal.

Este trabajo permitió poner en práctica todos los cambios propuestos en la enseñanza del diseño durante los últimos años, dio lugar también a la identificación de las falencias que aún se posee como carrera, permitiendo una reflexión sobre aquellos aspectos que se deben fortalecer en la enseñanza de la disciplina, tal es el caso de la mejora en los procesos investigativos conjuntos, el fortalecimiento del trabajo en equipo con docentes y estudiantes de otras áreas del conocimiento, la generación de metodologías especializadas resultados del trabajo continuo en proyectos similares, y sobre todo, la necesidad de enterrar de una vez la idea de que el diseñador es el materializador y embellecedor de las ideas de otros, sino que es un profesional valioso que 
respaldado en el conocimiento propio, en la vinculación con otras disciplinas, en la investigación profunda del ser humano y su contexto, y en su participación activa desde el inicio hasta el final de un proyecto, tiene la capacidad de generar estrategias y soluciones efectivas para problemáticas y necesidades de comunicación visual de cualquier índole.

A pesar de que el vínculo investigativo entre las carreras mencionadas no llegó a ser tan profundo como se esperaba, se generaron grandes avances, puesto que fue la primera vez que Diseño pudo visibilizarse a partir de un proyecto de titulación resultado de una investigación académica conjunta, culminando con el reconocimiento de los biólogos al aporte del Diseño Gráfico en los procesos educativos y de vinculación entre las comunidades objetivos y los profesionales participantes en el proyecto, además de la alta valoración que se le dio a la propuesta gráfica en relación a la efectividad que tuvo en relación al material educativo con el que trabajaban hasta el momento y el impacto que causó en el público objetivo.

Al momento el proyecto ganó el Fondo Publícalo 2019, a través del cual se le otorga al graduado un presupuesto para la producción e implementación del producto, el que se espera llegue a ejecutarse una vez las unidades educativas de Educación General Básica del Distrito Metropolitano de Quito retornen a la presencialidad.

\section{Conclusión}

En conclusión, la labor realizada en la enseñanza del diseño durante estos últimos años y los resultados obtenidos a partir de proyectos como el mencionado en este documento, son la evidencia de una nueva visión del diseño, y en relación con lo que menciona Kassim Vera, debemos empezar a construir críticas sensatas de los agentes que componen (o descomponen) la profesión, educación y escena del diseño (2017). Esto implica que el diseñador requiere repensar continuamente su profesión y su labor con el fin de adaptarse con efectividad a los requerimientos de las nuevas sociedades, y que mejor si estos procesos inician en las aulas, pues seguramente mientras más temprano se inserten estos modelos, más espacio disponible se tendrá en la academia para compartir la experiencia académica e investigativa y generar experiencias de aprendizaje significativas.

Respecto a la aplicación de estos nuevos procesos en la ejecución de los proyectos de titulación, se puede mencionar que el éxito de los estudiantes mencionados y otros que a causa de espacio no constan en este documento, se ha constituido en el referente y punto de ancla para las nuevas propuestas, buscando cada vez aportes mayores, pues los estudiantes se sienten motivados por las experiencias de sus compañeros, además de generarse un sentido de fidelización con la Carrera y la Universidad y de empatía con sus futuros colegas.

Desde la experiencia docente, es evidente el aprendizaje profundo que esta propuesta va dejando en las aulas y la forma en que se empieza a expandir hacia otras materias y en procesos de internacionalización mediante cátedra compartida, que se han propuesto con más fuerza a partir de las limitaciones generadas por la pandemia, convirtiendo con ello los obstáculos en grandes oportunidades. 


\section{Bibliografia y fuentes documentales}

Libros

Ander-Egg, E. (1991). El taller una alternativa de renovación pedagógica. (2da Ed.). Buenos Aires: Magisterio del Río de la Plata.

Belluccia, R. (2007). El diseño gráfico y su enseñanza: ilusiones y desengaños. Buenos Aires: Paidos.

Bürdek, B. (2003). «Tiempo de diseño». En B. E. Bürdek, El diseño estratégico. Barcelona: Editorial Gustavo Gili, SA.

Finkel, D. (2008). Dar clase con la boca cerrada. Barcelona: Publicaciones de la Universitat de Valéncia.

Forner, M. (2015). La enseñanza de proyectos de Diseño Gráfico. S.L.: Universidad Miguel Hernández

Frascara, J. (1997). Diseño Gráfico para la gente. Buenos Aires: Infinito.

Frascara, J. (2012). El diseño de comunicación. Buenos Aires: Infinito.

Frascara, J. (2017). Enseñando Diseño. Buenos Aires: Infinito.

Jones, C. (1985). Diseñar el diseño. Barcelona: Editorial Gustavo Gili S.A.

Litwin, E. (2012). El oficio de enseñar. Condiciones y contexto. Buenos Aires: Paidós.

Mazzeo, C. (2015). ¿Qué dice del diseño la enseñanza del diseño? Buenos Aires: Ediciones Infinito.

Mazzeo, C. (2017). Diseño y sistema. Bajo la punta del iceberg. Buenos Aires: Infinito.

Ricard, A. (1986). Hablando de diseño. Barcelona: Hogar del libro S.A.

Wong, W. (1979). Fundamentos del diseño bi y tridimensional. México: Gustavo Gili.

Artículos

Branda, S., Porta, L. (2012) «Maestros que marcan. Biografía personal e identidad profesional en docentes memorables». Profesorado. Revista de Currículum y Formación de Profesorado. Vol. 16 (No.3). [p. 231-p. 243] Recuperado de: https:// www.redalyc.org/pdf/567/56725002012.pdf

De santis Xifra, M., Yedaide, M. (2018). «Didáctica proyectual: un acercamiento documental a las configuraciones narrativas identitarias de cátedra en la FAUD, UNMDP». Praxis educativa. Vol. 22 (No. 1). [p. 13-p. 23]. Recuperado de: https://cerac.unlpam.edu.ar/index.php/praxis/article/view/2082

Novo, M. (2005). «Educación ambiental y Educación no formal: dos realidades que se realimentan». Revista de educación. Vol. (No. 338) [p. 145-p. 165]. DOI: 10.4438/1988-592X-0034-8082-RE

Rodríguez, R. (2016). "La pedagogía del diseño gráfico basada en la investigación en diseño». Revisión bibliográfica. ICONOFACTO. Vol. 12 (No. 19). [p. 254-p. 268]. DOI: http://dx.doi.org/10.18566/iconofact.v12.n19.a11

Tresserras. J. (2015). «Diseño e interdisciplinariedad. Una visión». Revistes Científiques de la Universitat de Barcelona. Vol 34. (N. 2), [p. 5-p. 18]. Recuperado de: https://revistes.ub.edu/index.php/waterfront/article/view/18829

Vera, K. (2017). «Crítica, política y diseño». Designaholic.mx. Recuperado de: http://designaholic.mx/2017/04/25663.HTML

Yedaide, M. (junio, 2016). "Narrativa y enseñanza de lo proyectual: Intermitencias». Trabajo presentado en III Jornadas de Investigadores en Educación de Encuentros de la Facultad de Humanidades / UNMDP Recuperado de: https://fh.mdp.edu. ar/encuentros/index.php/jie/3jie/paper/view/1357/714 